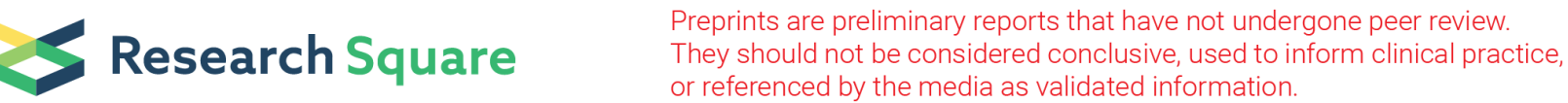

\section{Synthesis, Molecular Docking, And Antitumor Evaluation of Some New Pyrazole, Pyridine, And Thiazole Derivatives Incorporating Sulfonamide Residue}

\author{
Sameh Elgogary ( $\nabla$ selgogary@gmail.com ) \\ Jazan University \\ Ibrahim Radini \\ Jazan University
}

\section{Research Article}

Keywords: Pyrazoles, thiazoles, antitumor, sulfonamides

Posted Date: August 19th, 2021

DOI: https://doi.org/10.21203/rs.3.rs-792605/v1

License: (c) (1) This work is licensed under a Creative Commons Attribution 4.0 International License. Read Full License 


\section{Abstract}

Cancer is the second leading cause of death worldwide. There is always a huge demand for novel anticancer. New series of pyrazole, pyridine, thiazole derivatives containing sulfonamide moiety were prepared and screened for their antitumor activity against breast cancer cell line (MCF-7). The results of this investigation revealed that compounds $\mathbf{3}$ and $\mathbf{8}$ had significant anticancer activity against the MCF-7 cancer cell line with IC50 values 19.2 and $14.2 \mu \mathrm{M}$, respectively, in relation to the standard drug, doxorubicin.

\section{Introduction}

Cancer is one of the most dreadful diseases in the world and despite immense advances in the field of basic and clinical research, which have resulted in higher cure rates for a number of malignancies ${ }^{1,2}$. Tumor growth and metastasis depend on various factors, including the physiological process of angiogenesis. For example, elevated expression of vascular endothelial growth factor, epidermal growth factor, fibroblast growth factor, and platelet-derived growth factor are associated with tumor angiogenesis, metastases, survival, and resistance to apoptosis. Therefore, these growth factors have represented potential molecular targets for inhibition of tumor growth and progression ${ }^{3-6}$. Presently, a large number of anti-cancer drugs in clinical practice have shown pervasive side effects, low specificity, and multidrug resistance, so, there is always an urgent demand to develop novel anticancer drugs, and diverse new natural or synthetic compounds are developed continuously by scientists ${ }^{7}$. Sulfa drugs were the first broadly effective antibacterial to be used systemically and paved the way for the antibiotic revolution in medicine (see Fig. 1). In addition, Sulfonyl or sulfonamide hybrids were broadly explored for their anticancer activities via different mechanisms ${ }^{8-17}$ and it was found that they possess minimum side effects along with multi-drug resistance activity.

Therefore, and in continuation of our interest to develop a series of novel small molecules as potential antitumor agents that can block biologically relevant molecular targets ${ }^{18-22}$, we prepared certain pyrazoles, pyridines, thiazoles derivatives containing sulfonamide pharmacophores and studied their activity against breast cancer cell line (MCF-7).

\section{Results And Discussion}

\subsection{Chemistry Section}

Compounds $1 \mathrm{a}$ and $1 \mathrm{~b}$ were prepared according to literature procedure ${ }^{23}$. Condensation of $1 \mathrm{a}$ and $1 \mathrm{~b}$ and phenylhydrazine provided the hydrazones derivatives $\mathbf{2} \mathbf{a}$ and $\mathbf{2} \mathbf{b}$. The Vilsmeier-Haack reaction using 2.5 equiv of phosphoryl chloride performed a double addition of reagent to afford, ultimately after hydrolysis, the desired pyrazole 4-carbaldehyde derivative $\mathbf{3}$ (Scheme 1). The structure of $\mathbf{3}$ was established using both spectral and analytical analyses, in addition to X-ray analysis (Fig. 2). The IR spectrum of $\mathbf{3}$ showed 
absorption bands at $\mathrm{v}=1672(\mathrm{CHO}), 1633(\mathrm{~N}=\mathrm{CH}-)$, and 1342, $1153(\mathrm{SO} 2)$; while $1 \mathrm{HNMR}$ showed signals at $10.52(\mathrm{~s}, 1 \mathrm{H}, \mathrm{CHO})$ and $9.28(\mathrm{~s}, 1 \mathrm{H}$, pyrazole $\mathrm{H}-5)$ besides the rest of protons in its expected locations.

Treatment of the pyrazole carbaldehyde derivative $\mathbf{3}$ with some selected nitrogenous active reagents, namely (thiosemicarbazide, carbothidihydrazide, and cyanoacetohydrazide), afforded the condensed adduct $4, \mathbf{5}$, and $\mathbf{6}$ respectively without any observation of cyclized products. The versatile $a, \beta-$ unsaturated nitrile $\mathbf{7}$ is obtained upon treatment of $\mathbf{3}$ with ethyl cyanoacetate in the presence of few drops of triethylamine which was directly subjected to react with acetophenone in boiling ethanol containing a catalytic amount of ammonium acetate to afford 1,2 dihydropyridine derivatives $\mathbf{8}$. The structure of $\mathbf{8}$ was established by both spectral and analytical analysis, the infra-red spectrum of compound $\mathbf{8}$ showed absorption peaks $\mathrm{v}=3360(\mathrm{NH}), 2218(\mathrm{CN}), 1655(\mathrm{CO})$, in addition, the $1 \mathrm{H}$ NMR exhibited signals at $\delta=$ $12.54(\mathrm{NH}), 9.28(\mathrm{~s}, 1 \mathrm{H}$, pyrazole $\mathrm{H}-5)$, and 7.74 (pyridine $\mathrm{H}-5$ ), supported the suggested structure. Treatment of 3 with different aldehydes, namely acetophenone and 2- acetylthiophene, gave the corresponding chalcone derivatives $9 a$ and $9 b$. The $1 \mathrm{H}-\mathrm{NMR}$ of $9 \mathrm{a}$ (as a represented example) shows two doublet protons with $\mathrm{J}$ coupling $15.30 \mathrm{~Hz}$, indicating that the chalcone derivatives exist in E-configuration (Scheme 2).

The pyrazole derivative 10 was achieved in good yield, via the treatment of $9 \mathbf{a}$ with hydrazine hydrate in boiling ethanol.

Bromination of compounds $\mathbf{2} \mathbf{a}$ and $\mathbf{2} \mathbf{b}$ using bromine in acetic acid afforded the bis-dibromide derivatives $11 \mathrm{a}$ and $11 \mathrm{~b}$ as sole products in very high yields. The structures of $12 \mathrm{a}$ and $\mathbf{1 2 b}$ were established by both spectral and analytical analyses. The appearance of the singlet signal at $\delta=7.57 \mathrm{ppm}(\mathrm{CH})$ is supported the suggested structures.

The monobromo derivative 11a could be isolated as a side product, indicated that the mechanism of the reaction involving the formation of the monobromo as intermediate. Treatment of compounds with urea or $\mathrm{N}$-phenylurea afforded the thiazoles derivatives 13 a-d in good yields (see, scheme 3 and experimental section)

\subsection{Anticancer Activity Screening \\ 2.2.1. Cytotoxicity Assay}

The selectivity and sensitivity of malignancy and normal cells were checked by cytotoxicity assay of the experimental compounds. Ten of the newly synthesized compounds were examined for their anticancer action counter to MCF-7 cancer cell line through a high-throughput screening technique, and the results are furnished in Table 1. The screening of the $\mathrm{IC}_{50}$ results in Table 1 demonstrated that compound 8 revealed high anticancer activity against the MCF-7 cancer cell line with $\mathrm{IC}_{50}$ values $14.2 \mu \mathrm{M}$. Meanwhile, this compound has the topmost IC50 value, it can be estimated as a primary hit. Compounds 3, 13a, and $13 \mathrm{~b}$ exhibited high proliferative activity against the chosen cell line with $I C_{50}$ values 19.2, 28.1, and $35.1 \mu \mathrm{M}$, respectively. On the other hand, two Compounds (2a and 10) demonstrated trivial anticancer 
activity against the MCF-7 cell line with $\mathrm{IC}_{50}$ values 210.8 and $149.3 \mu \mathrm{M}$, respectively. From the previously stated study, we could realize that compounds 3 and 8 presented a good anticancer activity through all the established compounds expressed by their $I C_{50}$ values associate with doxorubicin $I C_{50}=1.19 \mu \mathrm{M}$. The biological profile of these compounds could be optimized by additional studies and could be used as lead compounds.

Table 1

Docking, binding energy calculations, and antitumor activity assay against MCF-7 of the newly synthesized compounds:

\begin{tabular}{|lllll|}
\hline $\begin{array}{l}\text { Comp. } \\
\text { No. }\end{array}$ & $\begin{array}{l}\text { MCF-7 IC50 } \\
(\mu \mathrm{M})\end{array}$ & $\begin{array}{l}\text { Binding Energy } \\
(\mathrm{kcal} / \mathrm{mol})\end{array}$ & $\begin{array}{l}\text { C-Docker } \\
\text { Energy }(-)\end{array}$ & $\begin{array}{l}\text { C-Docker Interaction } \\
\text { Energy }(-)\end{array}$ \\
\hline $\mathbf{2 a}$ & 149.2 & 11.2958 & 31.8572 & 53.8868 \\
\hline $\mathbf{2 b}$ & 88.2 & 9.5428 & 23.3008 & 57.2528 \\
\hline $\mathbf{3}$ & 19.2 & -10.32 & 10.2987 & 53.3724 \\
\hline $\mathbf{8}$ & 14.2 & -26.3 & 29.77 & 50.25 \\
\hline $\mathbf{9 a}$ & 89.6 & 10.7781 & 26.3799 & 73.4747 \\
\hline $\mathbf{1 0}$ & 210.8 & 15.6467 & 0.17243 & 64.7635 \\
\hline $\mathbf{1 3 a}$ & 28.1 & -5.8561 & 23.7322 & 43.0261 \\
\hline $\mathbf{1 3 b}$ & 35.1 & -3.3595 & 14.2812 & 44.1445 \\
\hline $\mathbf{1 3 c}$ & 78.3 & 3.6573 & 22.5583 & 44.5716 \\
\hline $13 d$ & 62.3 & 1.3936 & 19.0755 & 57.9311 \\
\hline
\end{tabular}

\subsubsection{Molecular Modeling}

Molecular docking for synthesized compounds was performed to explore their biological profile due to their binding with CDK2. Subsequently, their bound conformations and binding affinities were estimated. The structure preparation of the receptor was done by the removal of co-crystallized ligands and water molecules before energy minimization using the CHARMM force field. Hydrogens were added to the protein followed by adding the charges. The CDK2 coordinates were gained from the protein data bank (PDB 6GUH) and the structure was adjusted by Accelrys Discovery Studio 2.5. The missing residues were added as well as the structure was relaxed to correct the protein errors. Finally, the active site of CDK2 was distinct and all the synthesized compounds were docked and their binding energies were calculated. The synthesized derivatives were docked utilizing the default parameters of the C-Docker protocol (see Figures. 3 and 4), (Table 1).

\section{Materials And Methods}


A digital Gallen-KampMFB-595 instrument was used to measure melting points using open capillary tubes. The results were not corrected. KBr pellets were used to measure IR spectra using a Shimadzu FTIR-440 spectrometer. The MS-50 Kratos (A.E.I.) spectrometer, equipped with a data system, was used to obtain mass spectra at $70 \mathrm{eV}$. Chemical shifts in ppm units for ${ }^{1} \mathrm{H}$ NMR $(500 \mathrm{MHz})$ and ${ }^{13} \mathrm{CNMR}(125$ $\mathrm{MHz}$ ) were measured using a Bruker model Ultra Shield NMR spectrumrometer in DMSO-d6 using tetramethyl silane (TMS) as an internal reference. Cairo University's Microanalytical Center conducted the elemental studies (percent $\mathrm{C}, \mathrm{H}$, and $\mathrm{N}$ ). The appropriate precautions in handling moisture-sensitive compounds were taken. Solvents were dried by standard techniques. The monitoring of the progress of all reactions and homogeneity of the synthesized compounds were carried out and were run using thinlayer chromatography (TLC) aluminum sheets silica gel 60 F254 (Merck).

General procedure for synthesizing of hydrazones derivatives $\mathbf{2 a}$ and $\mathbf{2 b}$ : To $\mathbf{1 a}$ and $\mathbf{1 b}(10 \mathrm{mmol})$ in acetic acid $(100 \mathrm{~mL})$ and water $(10 \mathrm{~mL})$ phenylhydrazine $(11 \mathrm{mmol})$ was added. The reaction mixture was stirred at room temperature until completion (TLC, $5 \mathrm{~h}$ ). The solid which was formed was filtered off, washed with water, dried and crystallized from acetic acid to give:

4-[5-methyl-4-(1-(2-phenylhydrazineylidine) ethyl)-1H-1,2,3-triazol-1-yl]benzene-sulfomide (2a). Colorless crystals, yield 90\%, m.p. $=256-258^{\circ} \mathrm{C} . \mathrm{IR}\left(\mathrm{KBr}, \mathrm{cm}^{-1}\right) v=3323,3165\left(\mathrm{NH}_{2}\right), 3136(\mathrm{NH}), 1371,1141\left(\mathrm{SO}_{2}\right)$; ${ }^{1} \mathrm{H}$ NMR $\left(500 \mathrm{MHz}, \delta, \mathrm{pmm}, \mathrm{DMSO}-\mathrm{d}_{6}\right): \delta=9.35(\mathrm{~s}, 1 \mathrm{H}, \mathrm{NH}), 8.06(\mathrm{~d}, 2 \mathrm{H}, J=8.5 \mathrm{~Hz}, \mathrm{Ar}-\mathrm{H}), 7.88(\mathrm{~d}, 2 \mathrm{H}, J=$ $8.5 \mathrm{~Hz}, \mathrm{Ar}-\mathrm{H}), 7.60\left(\mathrm{~s}, 2 \mathrm{H}, \mathrm{NH}_{2}\right), 6.73-7.17(\mathrm{~m}, 5 \mathrm{H}, \mathrm{Ph}-), 2.62\left(\mathrm{~s}, 3 \mathrm{H}, \mathrm{CH}_{3}-\right), 2.43\left(\mathrm{~s}, 3 \mathrm{H}, \mathrm{CH}_{3}^{-}\right)$. Anal. Calcd. For. $\mathrm{C}_{17} \mathrm{H}_{18} \mathrm{~N}_{6} \mathrm{O}_{2} \mathrm{~S}$ (370.43): C, 55.12; $\mathrm{H}, 4.90 ; \mathrm{N}, 22.69$. Found: $\mathrm{C}, 55.20 ; \mathrm{H}, 4.80 ; \mathrm{N}, 22.80$.

N,N,-dimethyl-N'-[(4-(5-methyl-4-(1-(2-phenylhydrazineylidene)ethyl)-1H-1,2,3-triazol1yl)sulfonyl]formimidamide (2b). Colorless crystals, yield $88 \%$, m.p. $=179-180^{\circ} \mathrm{C} . \mathrm{IR}\left(\mathrm{KBr}_{\mathrm{cm}}{ }^{-1}\right) v=$ 3321, (NH), $1629(\mathrm{~N}=\mathrm{CH}-), 1373,1149\left(\mathrm{SO}_{2}\right) ;{ }^{1} \mathrm{H}$ NMR $\left(500 \mathrm{MHz}, \delta, \mathrm{pmm}, \mathrm{DMSO}-\mathrm{d}_{6}\right): \delta=9.33(\mathrm{~s}, 1 \mathrm{H}, \mathrm{NH})$, $8.29(\mathrm{~s}, 1 \mathrm{H}, \mathrm{N}=\mathrm{CH}$ ), 8.02 (d, 2H, J= $8.5 \mathrm{~Hz}, \mathrm{Ar}-\mathrm{H}), 7.82(\mathrm{~d}, 2 \mathrm{H}, J=8.5 \mathrm{~Hz}, \mathrm{Ar}-\mathrm{H}), 6.73-7.17(\mathrm{~m}, 5 \mathrm{H}, \mathrm{Ph}-)$, $3.16\left(\mathrm{~s}, 3 \mathrm{H}, \mathrm{N}-\mathrm{CH}_{3}\right), 2.94\left(\mathrm{~s}, 3 \mathrm{H}, \mathrm{N}-\mathrm{CH}_{3}\right) 2.63\left(\mathrm{~s}, 3 \mathrm{H}, \mathrm{CH}_{3}^{-}\right), 2.46\left(\mathrm{~s}, 3 \mathrm{H}, \mathrm{CH}_{3}^{-}\right) . \mathrm{MS}, \mathrm{m} / \mathrm{z}(\%): 425\left[\mathrm{M}^{+}\right](100)$. Anal. Calcd. For. $\mathrm{C}_{20} \mathrm{H}_{23} \mathrm{~N}_{7} \mathrm{O}_{2} \mathrm{~S}$ (425.51): C, 56.45; H, 5.45; N, 23.04. Found: C, 56.30; H, 5.60; N, 23.10.

$N^{\prime}-[(4-(4-(4-f o r m y l-1-p h e n y l-1 H-p y r a z o l-3-y l)-5-m e t h y l-1 H-1,2,3-t r i a z o l-1-y l)$ phenyl)sulfonyl]-N,Ndimethylformimidamide (3). Phosphorous oxychloride (25 mmol) was added to DMF $(100 \mathrm{~mL})$ at $0{ }^{\circ} \mathrm{C}$ and stirred for $30 \mathrm{~min}$. $\mathbf{2 a}$ or $\mathbf{2 b}(10 \mathrm{mmol})$ were added slowly to this mixture and stirred for $5 \mathrm{~h}$. The crude reaction was then quenched into water $(1 \mathrm{~L})$ and stirred for an additional $1 \mathrm{~h}$ the solid formed was separated, washed with water, dried, and crystallized from methanol to afford $\mathbf{3}$.

Colorless crystals, yield 88\%, m.p. $=250-251^{\circ} \mathrm{C} . \mathrm{IR}\left(\mathrm{KBr}, \mathrm{cm}^{-1}\right) \quad v=1672,(\mathrm{CO}), 1633(\mathrm{~N}=\mathrm{CH}-), 1342,1153$ $\left(\mathrm{SO}_{2}\right) ;{ }^{1} \mathrm{H}$ NMR $\left(500 \mathrm{MHz}, \delta, \mathrm{pmm}, \mathrm{DMSO}-\mathrm{d}_{6}\right): \delta=10.52(\mathrm{~s}, 1 \mathrm{H}, \mathrm{CHO}), 9.28(\mathrm{~s}, 1 \mathrm{H}$, pyrazole H-5), 8.02 (s, $1 \mathrm{H}, \mathrm{N}=\mathrm{CH}), 8.00(\mathrm{~d}, 2 \mathrm{H}, J=8.6 \mathrm{~Hz}, \mathrm{Ar}-\mathrm{H}), 7.82(\mathrm{~d}, 2 \mathrm{H}, J=8.6 \mathrm{~Hz}, \mathrm{Ar}-\mathrm{H}), 7.86-7.54(\mathrm{~m}, 5 \mathrm{H}, \mathrm{Ph}-), 3.14(\mathrm{~s}, 3 \mathrm{H}$, $\left.\mathrm{NCH}_{3}\right), 2.92\left(\mathrm{~s}, 3 \mathrm{H}, \mathrm{NCH}_{3}\right) 2.66\left(\mathrm{~s}, 3 \mathrm{H}, \mathrm{CH}_{3}-\right)$. MS, m/z (\%): $463\left[\mathrm{M}^{+}\right]$(100). Anal. Calcd. For. $\mathrm{C}_{22} \mathrm{H}_{21} \mathrm{~N}_{7} \mathrm{O}_{3} \mathrm{~S}$ (463.52): C, 57.01; H, 4.57; N, 21.15. Found: C, 57.20; H, 4.60; N, 21.10. 
General procedure for the synthesis of compounds 4, 5, and 6 . A solution of $3(4.63 \mathrm{~g}, 10 \mathrm{mmol})$ and thiosemecarbazide or carbothidihydrazide or cyanacetic acid hydrazide $(11 \mathrm{mmol})$ in a mixture of acetic acid/ ethanol ( 1:1) was refluxed for $6 \mathrm{~h}$. The solid formed on boiling was filtered of, dried and crystallized from acetic acid to afford:

2-[(3-(1-(4-(N-(dimethylamino) methylene) sulfamoyl) phenyl)-5-methyl-1H-1,2,3,-triazol-4-yl)-1-phenyl-1Hpyrazol-4-yl)methylenelhydrazine-1-carbothioamide (4). Pale yellow crystals in 86\% yield; m.p. 234-236 ${ }^{\circ} \mathrm{C}$. IR $\left(\mathrm{KBr}, \mathrm{cm}^{-1}\right) v=3427(\mathrm{NH}), 3329,3253\left(\mathrm{NH}_{2}\right), 1637(\mathrm{C}=\mathrm{N}), 1346,1143\left(\mathrm{SO}_{2}\right) ;{ }^{1} \mathrm{H} \mathrm{NMR}(500 \mathrm{MHz}, \delta$, pmm, DMSO-d $\left.{ }_{6}\right): \delta=11.55(\mathrm{~s}, 1 \mathrm{H}, \mathrm{NH}), 9.29(\mathrm{~s}, 1 \mathrm{H}$, pyrazole $\mathrm{H}-5), 8.74(\mathrm{~s}, 1 \mathrm{H}, \mathrm{CH}=\mathrm{N}), 8.30\left(\mathrm{~s}, 2 \mathrm{H}, \mathrm{NH}_{2}\right)$, $8.04(\mathrm{~d}, 2 \mathrm{H}, J=8.5 \mathrm{~Hz}, \mathrm{Ar}-\mathrm{H}), 7.91$ (d, 2H, J=8.5 Hz, Ar-H), 7.89 (s, 1H, N=CH), 7.59-7.37 ( m, 5H, Ph-H), $3.17\left(\mathrm{~s}, 3 \mathrm{H}, \mathrm{NCH}_{3}\right), 2.94\left(\mathrm{~s}, 3 \mathrm{H}, \mathrm{NCH}_{3}\right) 2.68\left(\mathrm{~s}, 3 \mathrm{H}, \mathrm{CH}_{3}-\right)$. Anal. Calcd. For. $\mathrm{C}_{23} \mathrm{H}_{24} \mathrm{~N}_{10} \mathrm{O}_{2} \mathrm{~S}_{2}$ (536.63): $\mathrm{C}$, 51.58; $H, 4.51 ; \mathrm{N}, 26.10$. Found: $C, 51.60 ; \mathrm{H}, 4.70 ; \mathrm{N}, 26.20$.

$N^{\prime}-[(4-(4-(4-(2-(h y d r a z i n e c a r b o t h i o y l) h y d r a z i n e y l i d e n e) m e t h y l)-1-p h e n y l-1 H-p y r a z o l-3 y l)-1 H--5-m e t h y l-1 H-$ 1,2,3-triazol-1-yl) phenyl)sulfonyl]-N,N,-dimethylformimidamine (5). Pale yellow crystals in $86 \%$ yield; m.p. 202-204 $\left.{ }^{\circ} \mathrm{C} . \mathrm{IR}\left(\mathrm{KBr}, \mathrm{cm}^{-1}\right) v=3232-3138\left(2 \mathrm{NH}, \mathrm{NH}_{2}\right)\right), 1633(\mathrm{C}=\mathrm{N}), 1338,1147\left(\mathrm{SO}_{2}\right) ;{ }^{1} \mathrm{H} \operatorname{NMR}(500$ $\mathrm{MHz}, \delta$, pmm, DMSO-d $\left.{ }_{6}\right): \delta=11.92$ (s., $\left.1 \mathrm{H}, \mathrm{NH}\right), 11.77\left(\mathrm{~s}, 2 \mathrm{H}, \mathrm{NH}_{2}\right), 9.92(\mathrm{~s}, 1 \mathrm{H}, \mathrm{NH}), 8.79(\mathrm{~s}, 1 \mathrm{H}$, pyrazole $\mathrm{H}-5), 8.29(\mathrm{~s}, 1 \mathrm{H}, \mathrm{CH}=\mathrm{N}), 8.04(\mathrm{~d}, 2 \mathrm{H}, J=8.5 \mathrm{~Hz}, \mathrm{Ar}-\mathrm{H}), 7.90(\mathrm{~d}, 2 \mathrm{H}, J=8.5 \mathrm{~Hz}, \mathrm{Ar}-\mathrm{H}), 7.87(\mathrm{~s}, 1 \mathrm{H}, \mathrm{N}=\mathrm{CH})$, 7.58-7.40 ( m, 5H, Ph-H), $3.17\left(\mathrm{~s}, 3 \mathrm{H}, \mathrm{NCH}_{3}\right), 2.94\left(\mathrm{~s}, 3 \mathrm{H}, \mathrm{NCH}_{3}\right) 2.69\left(\mathrm{~s}, 3 \mathrm{H}, \mathrm{CH}_{3}-\right)$. Anal. Calcd. For. $\mathrm{C}_{23} \mathrm{H}_{25} \mathrm{~N}_{11} \mathrm{O}_{2} \mathrm{~S}_{2}$ (551.65): C, 50.08; H, 4.57; N, 27.93. Found: C, 50.20; H, 4.70; N, 27.80.

$N^{\prime}-[(4-(4-(4-(2-(2-c y a n o a c e t y l)$ hydrazineylidene)methyl)-1-phenyl-1H-pyrazol-3-yl)-1H--5-methyl-1H-1,2,3triazol-1-yl) phenyl)sulfonyl]-N,N,-dimethylformimidamine (6). Colorless crystals in 79\% yield; m.p. 152$154{ }^{\circ} \mathrm{C} . \mathrm{IR}\left(\mathrm{KBr}, \mathrm{cm}^{-1}\right) \quad v=3217(\mathrm{NH}), 2250(\mathrm{CN}), 1697(\mathrm{CO}), 1627(\mathrm{C}=\mathrm{N})(\mathrm{CO}), 1344,1149\left(\mathrm{SO}_{2}\right) ;{ }^{1} \mathrm{H} \mathrm{NMR}$ $(500 \mathrm{MHz}, \delta$, pmm, DMSO-d 6 ): $\delta=11.81(\mathrm{~s} ., 1 \mathrm{H}, \mathrm{NH}), 9.10(\mathrm{~s}, 1 \mathrm{H}$, pyrazole $\mathrm{H}-5), 8.74(\mathrm{~s}, 1 \mathrm{H}, \mathrm{CH}=\mathrm{N}), 8.29$ $(\mathrm{s}, 1 \mathrm{H}, \mathrm{N}=\mathrm{CH}), 8.02(\mathrm{~d}, 2 \mathrm{H}, J=8.6 \mathrm{~Hz}, \mathrm{Ar}-\mathrm{H}), 7.89(\mathrm{~d}, 2 \mathrm{H}, J=8.6 \mathrm{~Hz}, \mathrm{Ar}-\mathrm{H}), 7.58-7.37(\mathrm{~m}, 5 \mathrm{H}, \mathrm{Ph}-\mathrm{H}), 4.25$ $\left(\mathrm{s}, 2 \mathrm{H}, \mathrm{CH}_{2}\right), 3.17\left(\mathrm{~s}, 3 \mathrm{H}, \mathrm{NCH}_{3}\right), 2.94\left(\mathrm{~s}, 3 \mathrm{H}, \mathrm{NCH}_{3}\right) 2.69\left(\mathrm{~s}, 3 \mathrm{H}, \mathrm{CH}_{3}\right)$. Anal. Calcd. For. $\mathrm{C}_{25} \mathrm{H}_{24} \mathrm{~N}_{9} \mathrm{O}_{3} \mathrm{~S}$ (530.17): C, 56.59; H, 4.56; N, 23.76. Found: C, 56.30; H, 4.50; N, 23.60.

Ethyl-2-cyano-3-[3-(1-(4-(N-(-dimethylamino) methylene) sulfamoyl) phenyl)-5-methyl-1 H-1,2,3-triazole-4-yl] acrylate (7). A solution of $3(10 \mathrm{mmol})$, ethyl cyanoacetate $(1.13 \mathrm{~mL}, 10 \mathrm{mmol})$ in the presence of 3 drops of triethylamine in absolute ethanol $(20 \mathrm{~mL})$ was refluxed for $5 \mathrm{~h}$. The solution was cooled and poured onto crushed ice. The resulting solid was filtered, dried and recrystallized from ethanol to yield 7. Yellow crystals in 90\% yield; m.p. $230-231^{\circ} \mathrm{C}$; IR (KBr, cm$\left.{ }^{-1}\right) v=2220$ (CN), 1703 (COOEt), 1624 (C = C), 1336, $1151\left(\mathrm{SO}_{2}\right) ;{ }^{1} \mathrm{H}$ NMR $(500 \mathrm{MHz}, \delta$, pmm, DMSO-d 6 ): $\delta=9.21(\mathrm{~s}, 1 \mathrm{H}$, pyrazole $\mathrm{H}-5), 8.17(\mathrm{~s}, 1 \mathrm{H}, \mathrm{N}=\mathrm{CH})$, $8.00(\mathrm{~s}, 1 \mathrm{H}, \mathrm{CH}=\mathrm{C}), 7.89$ (d, 2H, J= $8.6 \mathrm{~Hz}, \mathrm{Ar}-\mathrm{H}), 7.86(\mathrm{~d}, 2 \mathrm{H}, J=8.6 \mathrm{~Hz}, \mathrm{Ar}-\mathrm{H}), 7.56-7.45(\mathrm{~m}, 5 \mathrm{H}, \mathrm{Ph}-), 4.29$ (q, $2 \mathrm{H}, J=7.6 \mathrm{~Hz}, \mathrm{CH}_{2}$, ester), $3.13\left(\mathrm{~s}, 3 \mathrm{H}, \mathrm{NCH}_{3}\right), 2.92\left(\mathrm{~s}, 3 \mathrm{H}, \mathrm{NCH}_{3}\right) 2.66\left(\mathrm{~s}, 3 \mathrm{H}, \mathrm{CH}_{3}-\right), 1.27(\mathrm{t}, 3 \mathrm{H}, J=7.65$ $\mathrm{Hz}, \mathrm{CH}_{3}-$ ), MS, m/z (\%): $558\left[\mathrm{M}^{+}\right]$(100). Anal. Calcd. For. $\mathrm{C}_{27} \mathrm{H}_{26} \mathrm{~N}_{8} \mathrm{O}_{4} \mathrm{~S}$ (558.62): C, 58.05; H, 4.69; N, 20.06. Found: C, 58.20; $\mathrm{H}, 4.80 ; \mathrm{N}, 20.10$. 
$N^{\prime}-[(4-(4-(4-(3-c y a n o-2-o x o-6-p h e n y l-1,2-d i h y d r o p y r i d i n-4-y l)-1-p h e n y l-1 H-p y r a z o l-3-y l)-5-m e t h y l-1 H-1,2,3-$ triazol-1-yl)phenyl)sulfonyl]-N,N-dimethylformimidamide (8). A mixture of $4(2.79 \mathrm{~g}, 5 \mathrm{mmol})$, ammonium acetate $(2.73 \mathrm{~g}, 10 \mathrm{mmol})$ a acetophenone $(5 \mathrm{mmol})$ in absolute ethanol $(50 \mathrm{~mL})$ was refluxed for $12 \mathrm{~h}$. The solid which was precipitated during heating, was filtered, washed with water, dried, and crystallized from acetic acid to afford 8 as pale yellow crystals in $80 \%$ yield; m.p. $264-266^{\circ} \mathrm{C}$. IR $\left(\mathrm{KBr}_{\mathrm{cm}}{ }^{-1}\right) v=$ $3360(\mathrm{NH}), 2218(\mathrm{CN}), 1655$ (CO), 1337, $1154\left(\mathrm{SO}_{2}\right) ;{ }^{1} \mathrm{H}$ NMR (500 MHz, $\delta$, pmm, DMSO-d 6 ): $\delta=12.54$ ( brs., $1 \mathrm{H}, \mathrm{NH}$ ), $9.28(\mathrm{~s}, 1 \mathrm{H}$, pyrazole $\mathrm{H}-5), 8.12(\mathrm{~s}, 1 \mathrm{H}, \mathrm{N}=\mathrm{CH}), 8.00(\mathrm{~d}, 2 \mathrm{H}, J=8.6 \mathrm{~Hz}, \mathrm{Ar}-\mathrm{H}), 7.90(\mathrm{~d}, 2 \mathrm{H}, J=$ 8.6 Hz, Ar-H), $7.74(\mathrm{~s}, 1 \mathrm{H}$, pyridine $\mathrm{H}-5), 7.56-7.30(\mathrm{~m}, 10 \mathrm{H}, 2 \mathrm{Ph}-\mathrm{H}), 3.15\left(\mathrm{~s}, 3 \mathrm{H}, \mathrm{NCH}_{3}\right), 2.94\left(\mathrm{~s}, 3 \mathrm{H}, \mathrm{NCH}_{3}\right)$ 2.66(s, 3H, $\mathrm{CH}_{3}-$ ). Anal. Calcd. For. $\mathrm{C}_{33} \mathrm{H}_{27} \mathrm{~N}_{9} \mathrm{O}_{3} \mathrm{~S}$ (629.70): $\mathrm{C}, 62.94 ; \mathrm{H}, 4.32 ; \mathrm{N}, 20.02$. Found: $\mathrm{C}, 62.80 ; \mathrm{H}$, 4.50; N, 20.10.

General procedure for the synthesis of chalcones 9a-c: To a well-stirred solution of acetophenone or 2acetylthiophene or 2-acetylpyridine $3(10 \mathrm{mmol})$ in alcoholic $\mathrm{NaOH}(5 \%, 25 \mathrm{ml})$ at $0-5{ }^{\circ} \mathrm{C}$ a solution of 3 $(2.32 \mathrm{~g}, 5 \mathrm{mmol})$ was added gradually. Stirring was continued for $10 \mathrm{~h}$ at r.t. the resulting precipitate was filtrated, washed with water, dried, and crystallized from acetic acid to give.

N,N-dimethyl-N'-[(4-(5-methyl-4-(4-(3-oxo-3-phenylprop-1-en-1-yl)-1-phenyl-1H-pyrazol-3-yl)-1H-1,2,3-triazol1-yl)phenyl)sulfonyl]formimidamide (9a). Yellow crystals in $86 \%$ yield; m.p. $190-191^{\circ} \mathrm{C}$. IR $\left(\mathrm{KBr}, \mathrm{cm}^{-1}\right) v=$ $1678(\mathrm{CO}), 1631(\mathrm{C}=\mathrm{N}), 1597(\mathrm{C}=\mathrm{C}), 1340,1163\left(\mathrm{SO}_{2}\right) ;{ }^{1} \mathrm{H}$ NMR $\left(\mathrm{DMSO}-\mathrm{d}_{6}\right): \delta=9.49(\mathrm{~s}, 1 \mathrm{H}$, pyrazole H5), $8.60(\mathrm{~s}, 1 \mathrm{H}, \mathrm{CH}=\mathrm{N}), 8.10(\mathrm{~d}, 2 \mathrm{H}, J=8.5 \mathrm{~Hz}, \mathrm{Ar}-\mathrm{H}), 7.92(\mathrm{~d}, 2 \mathrm{H}, J=8.5 \mathrm{~Hz}, \mathrm{Ar}-\mathrm{H}), 7.80(\mathrm{~d}, 1 \mathrm{H}, \mathrm{J}=15.30 \mathrm{~Hz}$, $\mathrm{CH}=\mathrm{CH}), 7.70(\mathrm{~d}, 1 \mathrm{H}, \mathrm{J}=15.30 \mathrm{~Hz}, \mathrm{CH}=\mathrm{CH}), 7.59-7.45(\mathrm{~m}, 10 \mathrm{H}, 2 \mathrm{Ph}-\mathrm{H}), 3.13\left(\mathrm{~s}, 3 \mathrm{H}, \mathrm{NCH}_{3}\right), 2.94(\mathrm{~s}, 3 \mathrm{H}$, $\mathrm{NCH}_{3)} 2.67\left(\mathrm{~s}, 3 \mathrm{H}, \mathrm{CH}_{3}-\right.$ ). Anal. Calcd. For. $\mathrm{C}_{30} \mathrm{H}_{27} \mathrm{~N}_{7} \mathrm{O}_{3} \mathrm{~S}$ (565.65): $\mathrm{C}, 63.70 ; \mathrm{H}, 4.81 ; \mathrm{N}, 17.33$. Found: $\mathrm{C}$, $63.80 ; \mathrm{H}, 4.70 ; \mathrm{N}, 17.50$.

N,N-dimethyl-N'-[(4-(5-methyl-4-(4-(3-oxo-3-(thiophen - 2-yl) prop-1-en-1-yl)-1-phenyl-1 H-pyrazol-3-yl)-1H1,2,3-triazol-1-yl)phenyl)sulfonyl]formimidamide (9b). Yellow crystals in $80 \%$ yield; m.p. $166-168^{\circ} \mathrm{C}$. IR $\left(\mathrm{KBr}, \mathrm{cm}^{-1}\right) v=1643(\mathrm{CO}), 1631(\mathrm{C}=\mathrm{N}), 1597(\mathrm{C}=\mathrm{C}), 1342,1159\left(\mathrm{SO}_{2}\right) ;{ }^{1} \mathrm{H}$ NMR $\left(\mathrm{DMSO}-\mathrm{d}_{6}\right): \delta=9.49(\mathrm{~s}$, $1 \mathrm{H}$, pyrazole $\mathrm{H}-5), 8.60(\mathrm{~s}, 1 \mathrm{H}, \mathrm{CH}=\mathrm{N}), 8.48(\mathrm{~d}, 1 \mathrm{H}, J=16.70 \mathrm{~Hz}, \mathrm{CH}=\mathrm{CH}), 7.94(\mathrm{~d}, 2 \mathrm{H}, J=8.5 \mathrm{~Hz}, \mathrm{Ar}-\mathrm{H})$, $7.92(\mathrm{~d}, 2 \mathrm{H}, \mathrm{J}=8.5 \mathrm{~Hz}, \mathrm{Ar}-\mathrm{H}), 7.59-7.45(\mathrm{~m}, 9 \mathrm{H}, \mathrm{CH}=\mathrm{CH}, \mathrm{Ph}-\mathrm{H}, 3$ thiophene $-\mathrm{H})), 3.16\left(\mathrm{~s}, 3 \mathrm{H}, \mathrm{NCH}_{3}\right), 2.90(\mathrm{~s}$, $\left.3 \mathrm{H}, \mathrm{NCH}_{3}\right) 2.66$ (s, 3H, $\left.\mathrm{CH}_{3}-\right), \mathrm{MS}, \mathrm{m} / \mathrm{z}(\%): 571\left[\mathrm{M}^{+}\right]$(43). Anal. Calcd. For. $\mathrm{C}_{28} \mathrm{H}_{25} \mathrm{~N}_{7} \mathrm{O}_{3} \mathrm{~S}_{2}$ (571.67): $\mathrm{C}$, 58.83; $H, 4.41 ; N, 17.15$. Found: $C, 58.70 ; H, 4.60 ; N, 17.20$.

4-(4-(1',5-diphenyl-1'H,2H-[3,4'-bipyrazol]-3'-yl)-5-methyl-1H-1,2,3-triazol-1-yl)benzenesulfonamide (10). To a solution of appropriate chalcones $9 \mathrm{a}(2.78 \mathrm{~g}, 5 \mathrm{mmol})$ in ethanol $(30 \mathrm{ml})$, hydrazine hydrate $80 \%$ (12 $\mathrm{mmol}$ ) was added. The reaction mixture was refluxed for $6 \mathrm{~h}$. Left to cool to room temperature. The formed solid product was filtered, dried, and recrystallized from EtOH to afford 10 as colorless crystals in 82\% yield; m.p. $180-182^{\circ} \mathrm{C} . \mathrm{IR}\left(\mathrm{KBr}, \mathrm{cm}^{-1}\right) v=3218(\mathrm{NH}), 1631(\mathrm{C}=\mathrm{N}), 1597(\mathrm{C}=\mathrm{C}), 1340,1163\left(\mathrm{SO}_{2}\right) ;{ }^{1} \mathrm{H}$ NMR (DMSO-d $\left.\mathrm{d}_{6}\right): \delta=11.21(\mathrm{~s}, 1 \mathrm{H}, \mathrm{NH}), 9.49(\mathrm{~s}, 1 \mathrm{H}$, pyrazole $\mathrm{H}-5), 8.60(\mathrm{~s}, 1 \mathrm{H}, \mathrm{CH}=\mathrm{N}), 8.20(\mathrm{~s}, 1 \mathrm{H}$, pyrazoleH), $7.94(\mathrm{~d}, 2 \mathrm{H}, J=8.5 \mathrm{~Hz}, \mathrm{Ar}-\mathrm{H}), 7.92(\mathrm{~d}, 2 \mathrm{H}, J=8.5 \mathrm{~Hz}, \mathrm{Ar}-\mathrm{H}), 7.59-7.45(\mathrm{~m}, 10 \mathrm{H}, 2 \mathrm{Ph}-\mathrm{H})), 3.19(\mathrm{~s}, 3 \mathrm{H}$, 
$\left.\mathrm{NCH}_{3}\right), 2.92\left(\mathrm{~s}, 3 \mathrm{H}, \mathrm{NCH}_{3}\right) 2.65\left(\mathrm{~s}, 3 \mathrm{H}, \mathrm{CH}_{3}-\right.$ ). Anal. Calcd. For. $\mathrm{C}_{27} \mathrm{H}_{22} \mathrm{~N}_{8} \mathrm{O}_{2} \mathrm{~S}(522.16): \mathrm{C}, 62.06 ; \mathrm{H}, 4.24 ; \mathrm{N}$, 21.44. Found: $\mathrm{C}, 62.20 ; \mathrm{H}, 4.80 ; \mathrm{N}, 21.70$.

General procedure for the synthesis of compounds $11 \mathrm{a}, 12 \mathrm{a}$, and $12 \mathrm{~b}$. To a stirred cold solution of compound $1 \mathbf{a}$ or $1 \mathbf{b}(10 \mathrm{mmol})$ in acetic acid, bromine $(15 \mathrm{mmol})$ is add-drop wisely within $1 \mathrm{~h}$ and the stirring is continued at room temperature for another $4 \mathrm{hrs}$. The reaction mixture was heated at $80^{\circ} \mathrm{C}$ for another 6 hrs. The solid which was formed on heating filtered off, washed with ethanol ( $10 \mathrm{ml}$ ), dried, and crystallized from acetic acid to give :

4-[4-(2-bromoacetyl)-5-methyl-1H-1,2,3-triazol-1-yl] benzenesulfonamide (11 a). Colorless crystals, m.p. 230-232 ${ }^{\circ} \mathrm{C}$. IR $\left(\mathrm{KBr}, \mathrm{cm}^{-1}\right) v=3340,3194\left(\mathrm{NH}_{2}\right), 1681(\mathrm{C}=0), 1330,1161\left(\mathrm{SO}_{2}\right) .{ }^{1} \mathrm{H}-\mathrm{NMR}(500 \mathrm{MHz}, \delta$, pmm, DMSO-d $\mathrm{d}_{6}$ ): $\delta=8.01$ (d, 2H, J=8.6 Hz, Ar-H), 7.90 (d, 2H, J=8.6 Hz, Ar-H), $7.57\left(\mathrm{~s}, 2 \mathrm{H}, \mathrm{NH}_{2}\right.$ ), $4.66(\mathrm{~s}$, $\left.2 \mathrm{H}, \mathrm{CH}_{2}\right), 2.62\left(\mathrm{~s}, 3 \mathrm{H}, \mathrm{CH}_{3}\right)$. Anal. Calcd for $\mathrm{C}_{11} \mathrm{H}_{11} \mathrm{BrN}_{4} \mathrm{O}_{3} \mathrm{~S}$ (359.20): C, 36.78; $\mathrm{H}, 3.09 ; \mathrm{N}, 15.60$. Found $\mathrm{C}$, $36.90 ; \mathrm{H}, 3.20 ; \mathrm{N}, 15.70$.

4-[4-(2,2-dibromoacetyl)-5-methyl-1H-1,2,3-triazol-1-yl) benzene sulfonamide (12a). Colorless crystals in 90\% yield; m.p. $222-224{ }^{\circ} \mathrm{C}$. IR $\left(\mathrm{KBr}, \mathrm{cm}^{-1}\right) v=3226,3103\left(\mathrm{NH}_{2}\right), 1705(\mathrm{C}=0), 1338,1163\left(\mathrm{SO}_{2}\right) .{ }^{1} \mathrm{H}-\mathrm{NMR}$ $\left(500 \mathrm{MHz}, \delta, \mathrm{pmm}, \mathrm{DMSO}-\mathrm{d}_{6}\right): \delta=8.0(\mathrm{~d}, 2 \mathrm{H}, J=8.5 \mathrm{~Hz}, \mathrm{Ar}-\mathrm{H}), 7.92(\mathrm{~d}, 2 \mathrm{H}, J=8.5 \mathrm{~Hz}, \mathrm{Ar}-\mathrm{H}), 7.62(\mathrm{~s}, 2 \mathrm{H}$, $\left.\mathrm{NH}_{2}\right), 7.57(\mathrm{~s}, 1 \mathrm{H}, \mathrm{CH}), 2.62\left(\mathrm{~s}, 3 \mathrm{H}, \mathrm{CH}_{3}\right) .{ }^{13} \mathrm{C} \mathrm{NMR}\left(125 \mathrm{MHz}, \delta, \mathrm{pmm}, \mathrm{DMSO}-\mathrm{d}_{6}\right) \delta \mathrm{c} 10.09\left(\mathrm{CH}_{3}\right), 41.86$ $(\mathrm{CH}), 126.32,127.26,137.02(=\mathrm{CH}$ triazole), $137.42(=\mathrm{CH}$ triazole), 141.58, 145.56,181.32 (C=0). Anal. Calcd for $\mathrm{C}_{11} \mathrm{H}_{10} \mathrm{Br}_{2} \mathrm{~N}_{4} \mathrm{O}_{3} \mathrm{~S}$ (438.09): $\mathrm{C}, 30.16 ; \mathrm{H}, 2.30 ; \mathrm{N}, 12.79$. Found $\mathrm{C}, 30.10 ; \mathrm{H}, 2.40 ; \mathrm{N}, 12.90$.

$N$-[[4-[4-(2,2-dibromoacetyl)-5-methyl-1H-1,2,3-triazol-1-yl] phenyl] sulfonyl]-N, $N$-dimethylformimidamide (12b). Colorless crystals in 92\% yield; m.p. $214-216^{\circ} \mathrm{C}$. IR $\left(\mathrm{KBr}, \mathrm{cm}^{-1}\right) v=2991(\mathrm{CH}), 1699(\mathrm{C}=0), 1338$, $1151\left(\mathrm{SO}_{2}\right) .{ }^{1} \mathrm{H}-\mathrm{NMR}\left(500 \mathrm{MHz}, \delta, \mathrm{pmm}, \mathrm{DMSO}_{-} \mathrm{d}_{6}\right): \delta=8.28(\mathrm{~s}, 1 \mathrm{H}, \mathrm{CH}=\mathrm{N}), 8.02(\mathrm{~d}, 2 \mathrm{H}, J=8.6 \mathrm{~Hz}, \mathrm{Ar}-\mathrm{H})$, $7.86(\mathrm{~d}, 2 \mathrm{H}, J=8.6 \mathrm{~Hz}, \mathrm{Ar}-\mathrm{H}), 7.57(\mathrm{~s}, 1 \mathrm{H}, \mathrm{CH}), 3.16\left(\mathrm{~s}, 3 \mathrm{H}, \mathrm{NCH}_{3}\right), 2.93\left(\mathrm{~s}, 3 \mathrm{H}, \mathrm{NCH}_{3}\right), 2.62\left(\mathrm{~s}, 3 \mathrm{H}, \mathrm{CH}_{3}\right) .{ }^{13} \mathrm{C}$ $\operatorname{NMR}\left(125 \mathrm{MHz}, \delta\right.$, pmm, DMSO-d 6 ) $\delta \mathrm{c} 10.05\left(\mathrm{CH}_{3}\right), 35.20\left(\mathrm{NCH}_{3}\right), 41.04(\mathrm{CH}), 126.06,127.50,136.92(=$ $\mathrm{CH}$ triazole), 137.35 (= $\mathrm{CH}$ triazole), 141.59, 144.60, $160.16(\mathrm{~N}=\mathrm{C}), 181.31(\mathrm{C}=0) . \mathrm{MS}, \mathrm{m} / z(\%): 493[\mathrm{M}+]$ (56), $445\left[\mathrm{M}+-\mathrm{N}\left(\mathrm{CH}_{3}\right)_{2}\right]$ (4). Anal. Calcd for $\mathrm{C}_{14} \mathrm{H}_{15} \mathrm{Br}_{2} \mathrm{~N}_{5} \mathrm{O}_{3} \mathrm{~S}$ (493.17): C, 34.10; $\mathrm{H}, 3.07 ; \mathrm{N}, 14.20$. Found $\mathrm{C}$, $34.20 ; \mathrm{H}, 3.20 ; \mathrm{N}, 14.30$.

General procedure for the synthesis of compounds 13a-d: A solution of $12 \mathrm{a}$ or $12 \mathrm{~b}$ (10 mmol), and thioura or $N$-phenylurea in a mixture of acetic acid/ ethanol $(50 \mathrm{ml}, 1: 1)$ was refluxed for $10 \mathrm{~h}$. The solid formed on boiling was filtered of, dried and crystallized from acetone to afford:

4-[4-(2-aminothiazol-5-yl)-5-methyl-1 H-1,2,3-triazol-1-yl] benzene sulfonamide (13a). Pale yellow crystals, $83 \%$ yield m.p. $268-270^{\circ} \mathrm{C} . \mathrm{IR}\left(\mathrm{KBr}, \mathrm{cm}^{-1}\right) v=3329,3228\left(2 \mathrm{NH}_{2}\right), 2889(\mathrm{CH}), 1624(\mathrm{C}=\mathrm{N}), 1334,1155$ $\left(\mathrm{SO}_{2}\right) .{ }^{1} \mathrm{H}-\mathrm{NMR}\left(500 \mathrm{MHz}, \delta\right.$, pmm, DMSO-d 6 ): $\delta=8.66$ (brs., $2 \mathrm{H}, \mathrm{NH}_{2}$ ), 8.07 (d, 2H, J = 8.5 Hz, Ar-H), 7.90 $(\mathrm{d}, 2 \mathrm{H}, J=8.5 \mathrm{~Hz}, \mathrm{Ar}-\mathrm{H}), 7.88\left(\mathrm{~s}, 2 \mathrm{H}, \mathrm{NH}_{2}\right), 7.16(\mathrm{~s}, 1 \mathrm{H}$, thiazole- $\mathrm{H} 4), 2.52\left(\mathrm{~s}, 3 \mathrm{H}, \mathrm{CH}_{3}\right) \cdot{ }^{13} \mathrm{C} \mathrm{NMR}(125 \mathrm{MHz}$, $\delta$, pmm, DMSO-d 6 ) $\delta \mathrm{c}=9.99,103.89,125.82,127.31,130.11,133.07,135.47,137.62,145.20,169.99 . \mathrm{MS}$, 
$m / z(\%): 336[\mathrm{M}+]$ (22). Anal. Calcd for $\mathrm{C}_{12} \mathrm{H}_{12} \mathrm{~N}_{6} \mathrm{O}_{2} \mathrm{~S}$ (336.39): C, 42.85; $\mathrm{H}, 3.60 ; \mathrm{N}, 24.98$. Found C, 42.70; $H, 3.50 ; \mathrm{N}, 24.90$.

$N^{\prime}$-((4-(4-(2-aminothiazol-5-yl)-5-methyl-1H-1,2,3-triazol-1-yl)phenyl)sulfonyl)-N,N-dimethylformimidamide (13 b). Yellow crystals, 82\% yield, m.p. $234-236^{\circ} \mathrm{C}$. IR $\left(\mathrm{KBr}, \mathrm{cm}^{-1}\right) v=3354,3263\left(\mathrm{NH}_{2}\right), 2999(\mathrm{CH})$, 1635(C = N), 1340, $1143\left(\mathrm{SO}_{2}\right) .{ }^{1} \mathrm{H}-\mathrm{NMR}\left(500 \mathrm{MHz}, \delta, \mathrm{pmm}, \mathrm{DMSO}-\mathrm{d}_{6}\right): \delta=8.66\left(\mathrm{brs}, 2 \mathrm{H}, \mathrm{NH}_{2}\right), 8.28(\mathrm{~s}, 1 \mathrm{H}$, $\mathrm{CH}=\mathrm{N}), 8.01(\mathrm{~d}, 2 \mathrm{H}, J=8.5 \mathrm{~Hz}, \mathrm{Ar}-\mathrm{H}), 7.82(\mathrm{~d}, 2 \mathrm{H}, J=8.5 \mathrm{~Hz}, \mathrm{Ar}-\mathrm{H}), 7.14(\mathrm{~s}, 1 \mathrm{H}$, thiazole- H4), 3.16 (s, $\left.3 \mathrm{H}, \mathrm{NCH}_{3}\right), 2.93\left(\mathrm{~s}, 3 \mathrm{H}, \mathrm{NCH}_{3}\right), 2.52\left(\mathrm{~s}, 3 \mathrm{H}, \mathrm{CH}_{3}\right) .{ }^{13} \mathrm{C} \mathrm{NMR}\left(125 \mathrm{MHz}, \delta, \mathrm{pmm}, \mathrm{DMSO}-\mathrm{d}_{6}\right) \delta \mathrm{c}=9.9,103.9$, 125.5 (2C), 125.8, 127.2, 127.3 (2C), 130.1, 132.9, 135.4, 137.6, 144.8, 145.2, 169.90. Anal. Calcd for $\mathrm{C}_{15} \mathrm{H}_{17} \mathrm{~N}_{7} \mathrm{O}_{2} \mathrm{~S}_{2}$ (391.47): C, 46.02; $\mathrm{H}, 4.38 ; \mathrm{N}, 25.05$. Found $\mathrm{C}, 46.10 ; \mathrm{H}, 4.20 ; \mathrm{N}, 25.20$.

4-[5- methyl-4-(2-(phenylamino) thiazol-5-yl)- 1H-1,2,3-triazol-1-yl] benzene sulfonamide (13c). Yellow crystals $85 \%$ yield, m.p. $254-25^{\circ} \mathrm{C}$. IR $\left(\mathrm{KBr}, \mathrm{cm}^{-1}\right) v=3329,3242\left(\mathrm{NH}_{2}\right), 3147(\mathrm{NH}), 1618(\mathrm{C}=\mathrm{N}), 1338$, $1166\left(\mathrm{SO}_{2}\right) .{ }^{1} \mathrm{H}-\mathrm{NMR}\left(500 \mathrm{MHz}, \delta, \mathrm{pmm}, \mathrm{DMSO}-\mathrm{d}_{6}\right): \delta 10.51(\mathrm{~s}, 1 \mathrm{H}, \mathrm{NH}), 8.07(\mathrm{~d}, 2 \mathrm{H}, J=8.5 \mathrm{~Hz}, \mathrm{Ar}-\mathrm{H})$, $7.93(\mathrm{~d}, 2 \mathrm{H}, J=8.5 \mathrm{~Hz}, \mathrm{Ar}-\mathrm{H}), 7.65(\mathrm{~m}, 3 \mathrm{H}, \mathrm{Ph}-\mathrm{H}), 7.29(\mathrm{~s}, 1 \mathrm{H}$, thiazol $-\mathrm{H} 4), 7.20\left(\mathrm{~s}, 2 \mathrm{H}, \mathrm{NH}_{2}\right), 6.96(\mathrm{~m}, 2 \mathrm{H}$, $\mathrm{Ph}-\mathrm{H}), 2.58\left(\mathrm{~s}, 3 \mathrm{H}, \mathrm{CH}_{3}\right) .{ }^{13} \mathrm{C}$ NMR $(125 \mathrm{MHz}$, DMSO-d6) $\delta \mathrm{c}=10.1,48.6,92.4,104.2,117.3,121.9,125.6$ (2C), 127.2 (2C), 129.0, 133.0, 138.00, 138.2, 139.1, 140.5, 144.2, 162.6. Anal. Calcd for $\mathrm{C}_{18} \mathrm{H}_{16} \mathrm{~N}_{6} \mathrm{O}_{2} \mathrm{~S}_{2}$ (412.49): C, 52.41; H, 3.91; N, 20.37. Found C, 52.60; H, 3.60; N, 20.50.

N,N- dimethyl amino-N'-[(4-(5-methyl-4-(2-(phenylamino)thiazol-5-yl)- 1H-1,2,3-triazol-1-

yl)phenyl)sulfonyl]formimidamide (13 d). Pale yellow crystals, $78 \%$ yield, m.p. $265-267^{\circ} \mathrm{C}$. IR ( $\mathrm{KBr}, \mathrm{cm}^{-}$ $\left.{ }^{1}\right) v=3263(\mathrm{NH}), 1635(\mathrm{C}=\mathrm{N}), 1340,1143\left(\mathrm{SO}_{2}\right) .{ }^{1} \mathrm{H}-\mathrm{NMR}\left(500 \mathrm{MHz}, \delta, \mathrm{pmm}, \mathrm{DMSO}-\mathrm{d}_{6}\right): \delta=10.36(\mathrm{~s}, 1 \mathrm{H}$, $\mathrm{NH}), 8.28(\mathrm{~s}, 1 \mathrm{H}, \mathrm{CH}=\mathrm{N}), 8.01(\mathrm{~d}, 2 \mathrm{H}, J=8.5 \mathrm{~Hz}, \mathrm{Ar}-\mathrm{H}), 7.84(\mathrm{~d}, 2 \mathrm{H}, J=8.5 \mathrm{~Hz}, \mathrm{Ar}-\mathrm{H}), 7.67(\mathrm{~s}, 1 \mathrm{H}$, thiazole$\mathrm{H} 4), 7.28-6.93(\mathrm{~m}, 5 \mathrm{H}, \mathrm{Ph}-\mathrm{H}), 3.16\left(\mathrm{~s}, 3 \mathrm{H}, \mathrm{NCH}_{3}\right), 2.93\left(\mathrm{~s}, 3 \mathrm{H}, \mathrm{NCH}_{3}\right), 2.69\left(\mathrm{~s}, 3 \mathrm{H}, \mathrm{CH}_{3}\right) .{ }^{13} \mathrm{C} \mathrm{NMR}(125 \mathrm{MHz}$, DMSO-d6) $\delta c=10.1,35.2,41.2,92.37,104.2,117.0,125.6(2 \mathrm{C}), 125.7$ (2C), $127.5(2 \mathrm{C}), 129.0,129.1$, 139.82, 140.5, 141.1, 142.2, 160.2, 162.6, 163.8. Anal. Calcd for $\mathrm{C}_{21} \mathrm{H}_{21} \mathrm{~N}_{7} \mathrm{O}_{2} \mathrm{~S}(467.57)$ : C, 53.95; $\mathrm{H}, 4.53$; N, 20.97. Found C, 53.80; H, 4.70; N, 20.80.

\section{X-ray crystallography of compound 3.}

A single crystal of compound $\mathbf{3}$ was obtained by slow evaporation at room temperature, from dimethylformamide (DMF). The crystal structure was solved and refined using MaXus (Bruker Nonius, Deflt and Mac Science, Japan $)^{24}$. Mo Ka radiation $(\lambda=0.71073 \AA$ ) and a graphite monochromator were used for data collection. The chemical formula and ring labeling system are shown in Fig. 2. Crystal data for compound 3: $\mathrm{C}_{22} \mathrm{H}_{21} \mathrm{~N}_{7} \mathrm{O}_{3} \mathrm{~S}, \mathrm{M}_{\mathrm{r}}=463.520$; system, Monoclinic; space group, $\mathrm{P} 2{ }_{1} / \mathrm{c} ; \mathrm{a}=11.3957$ (3) $\AA$; $b=13.1843(5) \AA ; c=5.1206(5) \AA ; a=90.00^{\circ} ; \beta=106.413(3)^{\circ} ; \gamma=90.00^{\circ} ; V=2179.21(12) \AA^{3} ; Z=4 ; D_{x}=$ $1.413 \mathrm{Mg} \mathrm{m}^{-3}$; range for data $\theta=2.910-27.485^{\circ} ;(\mathrm{Mo}-\mathrm{Ka}) \mu=0.190 \mathrm{~mm}^{-1} ; \mathrm{T}=298 \mathrm{~K} ; 16702$ measured reflections; 4921 independent reflections; 2336 observed reflections; $R(a l l)=0.1595 ; R(g t)=0.0623$; $\mathrm{wR}($ ref $)=0.2021 ; \mathrm{wR}(\mathrm{gt})=0.1604 ; \mathrm{S}(\mathrm{ref})=0.995 ; 4921$ reflections; $\delta / \sigma_{\max }=0.004 ; \delta \rho_{\max }=0.278 \mathrm{e} \AA^{3} ; \delta \rho_{\min }$ 
$=-0.346 \mathrm{e} \AA^{3}$. Crystallographic data for the structure 5 have been deposited with the Cambridge Crystallographic Data Center (CCDC) under the number 1574927. Copies of the data can be obtained, free of charge, on application to CCDC 12 Union Road, Cambridge CB2 1EZ, UK [Fax: +44-1223-336033; email: deposit@ccdc.cam.ac.uk or at www.ccdc.cam.ac.uk.

\section{Molecular docking studies}

\section{Materials and methods}

Three-dimensional structure of human cycline-dependantkinase CDK2, PDB:2FVD, was obtained from protein data bank. Energy minimization of newly designed compounds was done by employing discovery studio 2.5 for structure refinement. The geometry of all designed analogues is typed with CHARMm ${ }^{25}$ force field; then partial charges are calculated by Momany Rone method ${ }^{26}$. Further, they are optimized through a smart minimizer algorithm, which performs 1000 steps of steepest descent with a root meansquare (RMS) gradient tolerance of 0.1 . Same as the preparation of ligands for the target, its active site was also passed with the energy minimization process and it was done using charm force field which is defined by the equation given below:

where $E=$ total energy, $E_{\mathrm{b}}=$ bond potential energy, $E_{\mathrm{q}}$ and $E_{\mathrm{f}}=$ bond angle potential energy, $E_{\mathrm{w}}=$ torsion energy, $E_{\mathrm{vdw}}=$ van der Waals interaction energy, $E_{\mathrm{el}}=$ electrostatic potential energy, $E_{\mathrm{hb}}=$ hydrogen bond energy, $E_{\mathrm{cr}}=$ energy constraints, and $E \mathrm{cj}=$ energy function ${ }^{25}$.

CDOCKER (CHARMm-based DOCKER), a docking program provided by discovery studio 2.5 , uses a CHARMm based molecular dynamics (MD) scheme to dock ligands into a receptor binding site, and then random conformations will be produced using high-temperature molecular dynamics. When these conformations are translated to the active site, candidate poses are then generated using random rigid body rotations followed by simulated annealing. CDOCKER offers all the advantages of full ligand flexibility (including bonds, angles, and dihedrals) and reasonable computation times. CDOCKER uses soft core potentials, which are found to be effective in exploring the conformational space of macromolecules used in various docking studies. The nonbonded interactions which involve van der Waals (vdW) and electrostatics are softened at different levels, except during the final minimization step ${ }^{27}$. Initially, ten conformations for each inhibitor are generated in the active site of the target enzyme, which is created as a spherical region with a diameter of $10 \AA$. Simulated annealing is performed using a flexible ligand and a rigid protein. Receptor-ligand interactions are calculated from grid extension 8.0, random conformations are generated using specific molecular dynamics steps, and the system is heated to $700 \mathrm{~K}$ in 2000 steps, cooling steps to 5000 , and cooling temperature to $300 \mathrm{~K}$. The final refinement step of minimization is performed using full potential. Minimized docking poses are then clustered, based on a heavy atom RMSD approach. The ranking is based on the total docking energy, which is composed of the ligand's intramolecular energy and the ligand-receptor interaction.

\section{Declarations}




\section{Author contributions}

Authors participated in the idea of this research and they carried out the synthesis, purification and characterization of all compounds by the different spectroscopic techniques. Both authors have read and approved he final version submitted

\section{Competing interests}

The authors declare no competing interests.

\section{References}

1. Eckhardt, S. Recent progress in the development of anticancer agents. Curr. Med. Chem, 2 (3), 419439 (2002).

2. Lee, C. W. et al. A novel stereo-selective sulfonylurea, 1-[1-(4-aminobenzoyl)-2,3-dihydro-1 H-indol-6sulfonyl]-4-phenyl-imidazolidin-2-one, has antitumor efficacy in in vitro and in vivo tumor models. Biochem. Pharmacol, 64 (3), 473-480 (2002).

3. Jayson, G. C. \& Hasan, J. VEGF antagonists. Expert Opin. Biol. Ther, 1 (4), 703-718 (2001).

4. Hamby, J. M. \& Showalter, H. D. H. Small Molecule Inhibitors of Tumor-Promoted Angiogenesis, Including Protein Tyrosine Kinase Inhibitors. Pharmacol. Ther, 82 (2), 169-193 (1999).

5. Aggarwal, B. B., Danda, D., Gupta, S. \& Gehlot, P. Models for prevention and treatment of cancer: Problems vs promises. Biochem. Pharmacol, 78 (9), 1083-1094 (2009).

6. Csermely, P., Ágoston, V. \& Pongor, S. The efficiency of multi-target drugs: the network approach might help drug design. Trends Pharmacol. Sci, 26 (4), 178-182 (2005).

7. Rojo, F., Albanell, J., Rovira, A., Corominas, J. M. \& Manzarbeitia, F. Targeted therapies in breast cancer. Semin. Diagn. Pathol, 25 (4), 245-261 (2008).

8. Andrea, S., Takashi, O., Antonio, M. \& Claudiu, T. S. Anticancer and Antiviral Sulfonamides. Curr. Med. Chem, 10 (11), 925-953 (2003).

9. Supuran, C. T. Carbonic anhydrases: novel therapeutic applications for inhibitors and activators. Nat. Rev. Drug Discovery, 7 (2), 168-181 (2008).

10. Neri, D. \& Supuran, C. T. Interfering with $\mathrm{pH}$ regulation in tumours as a therapeutic strategy. Nat. Rev. Drug Discovery, 10 (10), 767-777 (2011).

11. Minakuchi, T., Nishimori, I., Vullo, D., Scozzafava, A. \& Supuran, C. T. Molecular Cloning, Characterization, and Inhibition Studies of the Rv1284 $\beta$-Carbonic Anhydrase from Mycobacterium tuberculosis with Sulfonamides and a Sulfamate. J. Med. Chem, 52 (8), 2226-2232 (2009).

12. Dubois, L. et al. Imaging of CA IX with fluorescent labelled sulfonamides distinguishes hypoxic and (re)-oxygenated cells in a xenograft tumour model. Radiother. Oncol, 92 (3), 423-428 (2009).

13. Gitto, R. et al. Identification of 3,4-Dihydroisoquinoline-2(1H)-sulfonamides as Potent Carbonic Anhydrase Inhibitors: Synthesis, Biological Evaluation, and Enzyme - Ligand X-ray Studies. J. Med. 
Chem, 53 (6), 2401-2408 (2010).

14. Huang, Z., Lin, Z. \& Huang, J. A novel kind of antitumour drugs using sulfonamide as parent compound. Eur. J. Med. Chem, 36 (11), 863-872 (2001).

15. Alafeefy, A. M., Kadi, A. A., Al-Deeb, O. A. \& El-Tahir, K. E. H. Al-jaber, N. A., Synthesis, analgesic and anti-inflammatory evaluation of some novel quinazoline derivatives. Eur. J. Med. Chem, 45 (11), 4947-4952 (2010).

16. Rakesh, K. P. et al. Recent Development of Sulfonyl or Sulfonamide Hybrids as Potential Anticancer Agents: A Key Review. Anticancer Agents Med. Chem, 18 (4), 488-505 (2018).

17. Xing, N. et al. Design, Synthesis, and Antitumor Activity of a Series of Novel 4-(Aromatic Sulfonyl)-1oxa-4-azaspiro[4.5]deca-6,9-dien-8-ones., 25 (22), 5459 (2020).

18. Radini, A. M., Khidre, I. E. \& El-Telbani, R. M. E., Synthesis and antimicrobial evaluation of new pyrazoline and pyrazolinyl thiazole derivatives bearing tetrazolo [1, 5-a] quinoline moiety. Lett. Drug Des. Discovery, 13 (9), 921-931 (2016).

19. Khidre, R. E. \& Radini, I. A. M. Synthesis and Antimicrobial Activity of Novel Heterocycles Utilizing 3(1,4-Dioxo-3,4-dihydrophthalazin-2(1H)-yl)-3-oxopropanenitrile as Precursors. J. Heterocycl. Chem, 56 (3), 850-858 (2019).

20. Khidre, R. E., Radini, I. A. M. \& Ibrahim, D. Synthesis of a novel heterocyclic scaffold utilizing 2-cyano$\mathrm{N}$-(3-cyano-4, 6-dimethyl-2-oxopyridin-1-yl) acetamide. ARKIVOC, 301-317(2016).

21. Khidre, R. E. \& Radini, I. A. M. Design, synthesis and docking studies of novel thiazole derivatives incorporating pyridine moiety and assessment as antimicrobial agents. Sci. Rep, 11 (1), 7846 (2021).

22. Radini, I. A. M., Elsheikh, T. M., El-Telbani, E. M. \& Khidre, R. E. J. M. New potential antimalarial agents: design, synthesis and biological evaluation of some novel quinoline derivatives as antimalarial agents., 21 (7), 909 (2016).

23. Elgogary, S. R., Khidre, R. E. \& El-Telbani, E. M. Regioselective synthesis and evaluation of novel sulfonamide 1,2,3-triazole derivatives as antitumor agents. J. Iran. Chem. Soc, 17 (4), 765-776 (2020).

24. Mackay, S., Gilmore, C., Edwards, C., Stewart, N. \& Shankland, K. MaXus computer program for the solution and refinement of crystal structures. Bruker Nonius, The Netherlands, MacScience, Japan \& The University of Glasgow(1999).

25. Brooks, B. R. et al. o. c. c., CHARMM: the biomolecular simulation program. J. Comput. Chem, 30 (10), 1545-1614 (2009).

26. Momany, F. A. \& Rone, R. Validation of the general purpose QUANTA ${ }^{\circledR} 3.2 /$ CHARMm ${ }^{\circledR}$ force field. J. Comput. Chem, 13 (7), 888-900 (1992).

27. Wu, G., Robertson, D. H., Brooks, C. L. III \& Vieth, M. Detailed analysis of grid-based molecular docking: A case study of CDOCKER-A CHARMm-based MD docking algorithm. J. Comput. Chem, 24 (13), 1549-1562 (2003). 
Figures<smiles>Nc1ccc(S(N)(=O)=O)cc1</smiles>

Sulphonamide

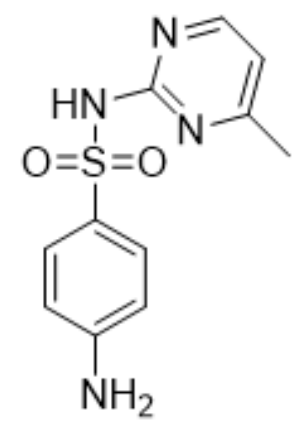

Sulfamerazine

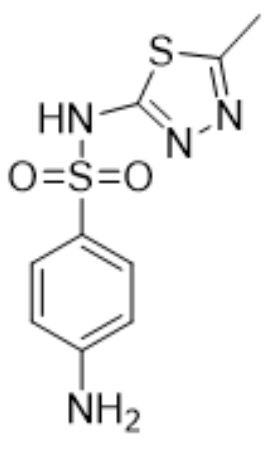

Sulfathiazole

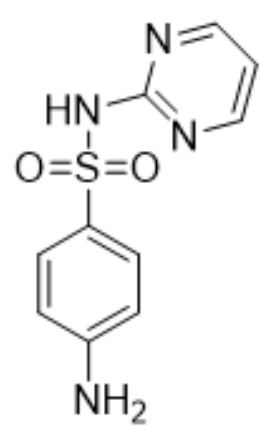

Sulfadiazine

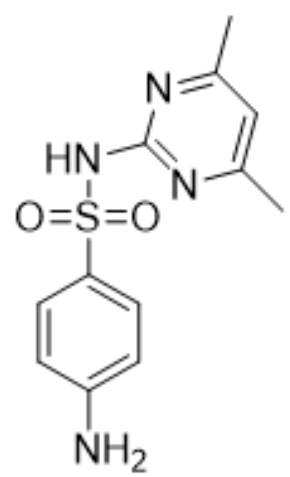

Sulfamethazine<smiles>Cc1noc(NS(=O)(=O)c2ccc(N)cc2)c1C</smiles>

Sulfasoxazole

\section{Figure 1}

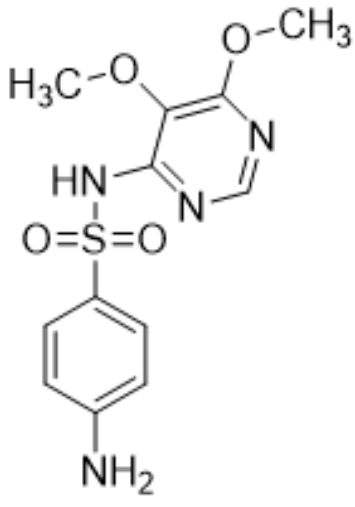

Sulfadimethoxine

Chemical structure of sulfonamides (SAs). 


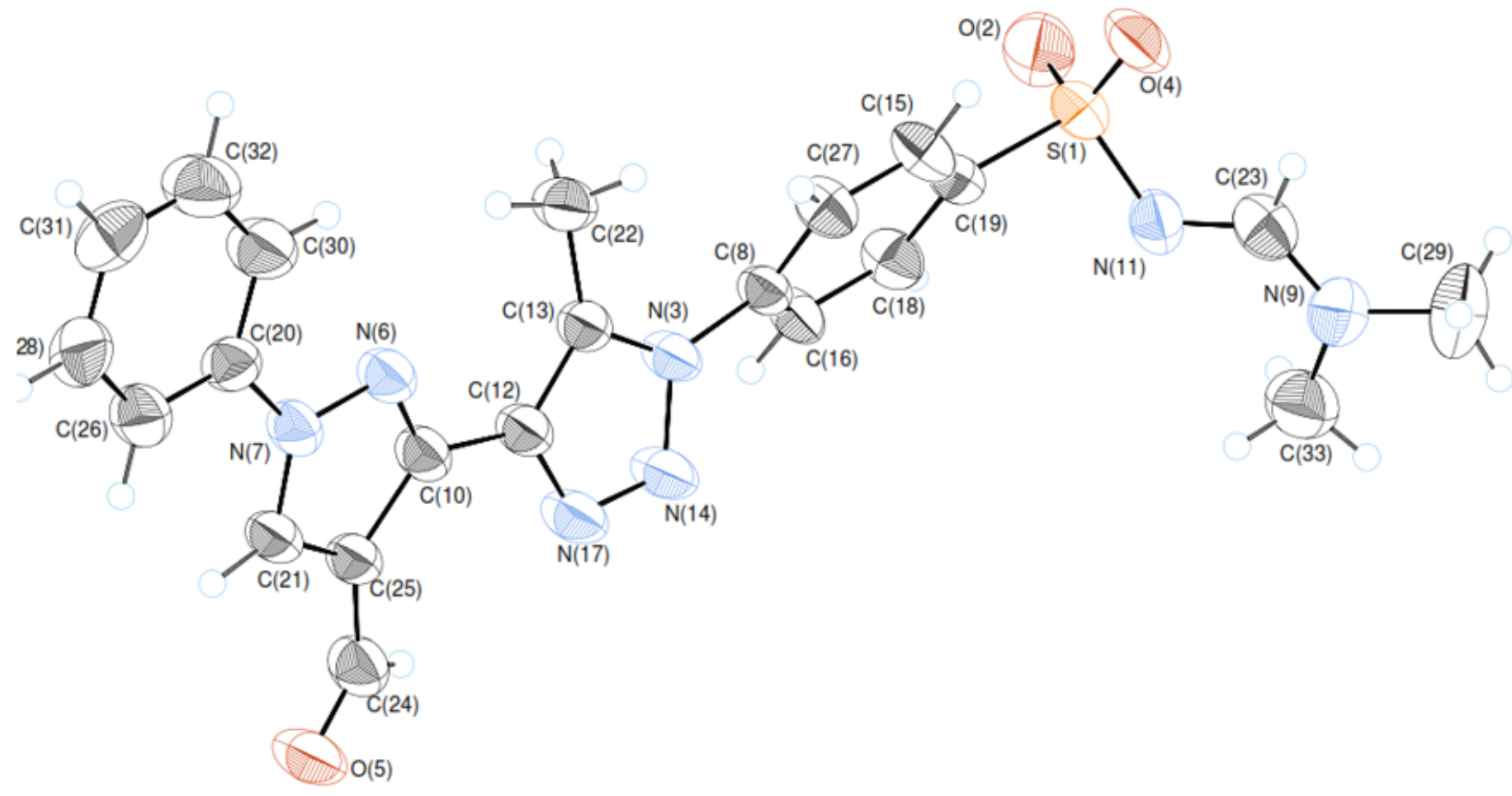

Figure 2

X-ray structure of 3 .

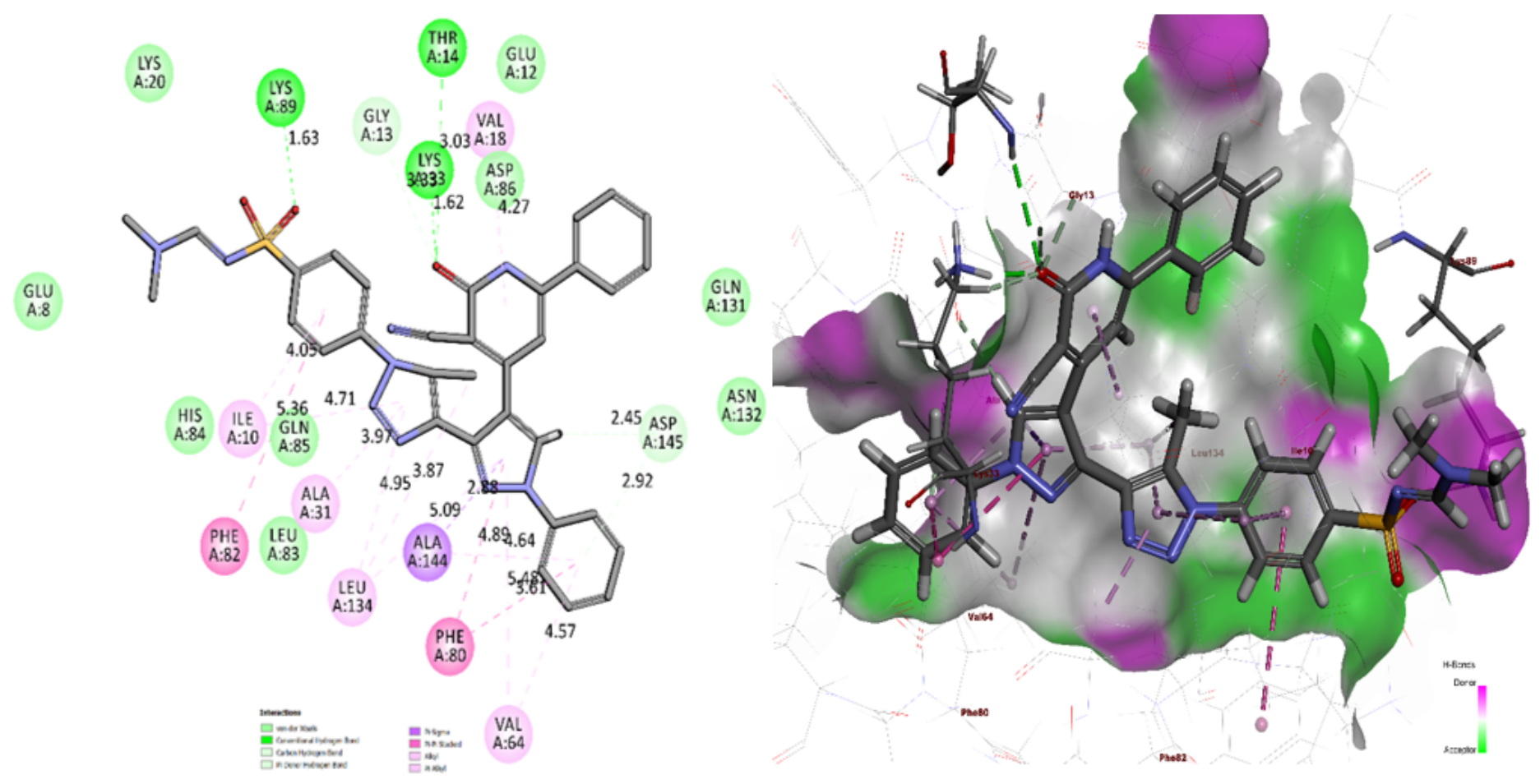

Figure 3 
Hydrogen bond interactions of compound 8 with the active site residues. It forms 3 HB with Lys33, Thr14, and Lys 89 .
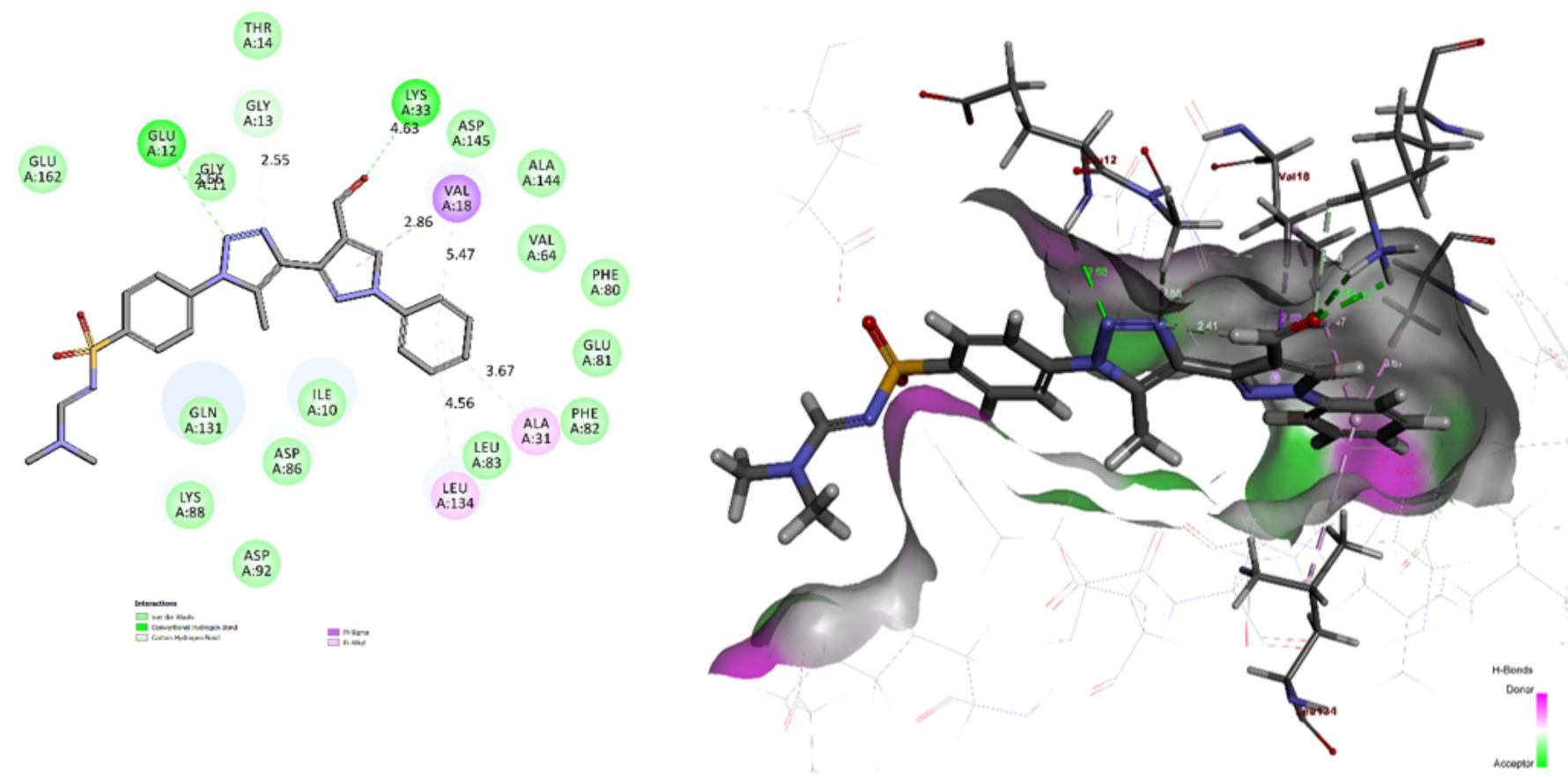

Figure 4

Hydrogen bond interactions of compound 3 with the active site residues of CDK2. It forms $1 \mathrm{HB}$ with Lys33.

\section{Supplementary Files}

This is a list of supplementary files associated with this preprint. Click to download.

- SupportingInformationScientificReports.pdf

- Scheme1.png

- Scheme2.png

- Scheme3.png 